\title{
MEMS Seismic Sensor with FPAA Based Interface Circuit for Frequency-Drift Compensation Using ANN
}

\author{
Ramesh Pawase ${ }^{1}$, N. P. Futane ${ }^{2}$ \\ ${ }^{1}$ Department of E\&TC, SITRC Nashik, Amrutvahini CoE, Sangamner, Savitribai Phule Pune University, 422608, +91- \\ 9766501804, India \\ ${ }^{2}$ Department of E\&TC, Government College of Engineering, Avsari, Savitribai Phule Pune University, 412405, +91- \\ 9004828465, India
}

\begin{tabular}{l}
\hline \hline Article Info \\
\hline Article history: \\
Received Apr 1, 2017 \\
Revised May 21, 2017 \\
Accepted Jun 7, 2017 \\
\hline
\end{tabular}

Keywords:

Analog signal processing Artificial Neural Network (ANN)

FPAA

MEMS

Seismic sensor

\begin{abstract}
Electrochemical MEMS seismic sensor is limited by its non-ideality of frequency dependent characteristics hence interface circuits for compensation is necessary. The conventional compensation circuits are limited by high power consumption, bulky external hardware circuitry. In these methods digital circuits are also limited by inherent analog to digital conversion and vice versa which consumes significant power, acquires more size and limits speed. A Field programmable analog array (FPAA) overcomes these limitations and gives fast, simple and user friendly development platform with less development speed comparable to ASIC. Recently FPAA becoming popular for rapid prototyping. The proposed system presents FPAA (Anadigm AN231E04) based hardware implementation of ANN model. Using this FPAA based compensation circuit, the error in frequency drift have been minimized in the range of $3.68 \%$ to about $0.64 \%$ as compared to ANN simulated results in the range of $23.07 \%$ to $0.99 \%$. This single neuron consumes of power of $206.62 \mathrm{~mW}$. and has minimum block wise resource utilization. The proposed hardware uses all analog blocks which remove the requirement of $\mathrm{ADC}$ and $\mathrm{DAC}$ reducing significant power and size of interface circuit. This work gives the SMART MEMS seismic sensor with reliable output and ANN based intelligent interface circuit implemented in FPAA hardware.
\end{abstract}

Copyright $\odot 2017$ Institute of Advanced Engineering and Science. All rights reserved.

\section{Corresponding Author:}

Ramesh Pawase,

Department of E\&TC, SITRC Nashik, Amrutvahini CoE, Sangamner,

Savitribai Phule Pune University, 422608, +91-9766501804, India.

Email: rameshpawase@gmail.com

\section{INTRODUCTION}

Information systems getting closer to physical world which are generating new opportunities in the area of sensing and controlling environment and different devices. In order to exploit these available opportunities, miniaturized information systems needs must be developed. Developing miniature sensing system and making it reliable is a driving force for development of Micro-Electro Mechanical Systems (MEMS), which construct both mechanical and electrical components in miniature in size. As MEMS technology is supplementary to Integrated Circuits technology, the synergy between mechanical, electrical and electronic system makes it breakthrough technology. MEMS devices have application in areas ranging from automobile and telecommunication switching to printers and inertial guidance system. MEMS are widely used in various sensing and actuators applications [1]. In this paper, it has been focused on FPAA based design and development of interface circuit for MEMS Seismic Sensor (MSS) output compensation. In these types of applications the FPAA offers better solution as rapid implementation is possible. The targeted commercially available FPAA- an Anadigm AN231K04 is an integrated circuit, containing circuits from 
combination of very small capacitors and transistors. Recently several vendors including Anadigm, provides service for creating an ASIC for user-defined application by configuring the FPAA. Development speed is time required to configure an FPAA is far less than that required to design, simulate and fabricate the ASIC. The accurate and stable design is possible due to use of switched capacitor-based analog design in FPAA, which minimizes offsets, nonlinear behavior, and component tolerances for long time with wide range of operating temperatures. The Anadigm FPAA Designer 2 tool has been used to define the architecture of Anadigm AN231K04 FPAA chips.

The major contributions of this paper are as follows:

a. ANN based compensation is applied for MEMS seismic sensor with minimum error.

b. FPAA based hardware implementation utilizing minimum resources and thirdly achieving minimum error using hardware implementation.

This paper is organized in following different sections. Second section deals with literature survey and gaps in existing work along with scope for the research work. Third Section discusses for MEMS Seismic Sensor (MSS) non-idealities and proposed work to compensate the non-idealities present in output of MSS. Section 4 gives implementation of artificial neural network based frequency effect compensation scheme using neuron model. Section 5 discusses on interface circuit implementation using FPAA. Results are discussed and analyzed in Section 6.

\section{RELATED WORK}

MEMS based Electrochemical Seismic sensor device characterization performed on a vibration table recorded in [2] It has been reported overall linear relationship between applied velocities and output voltage amplitudes with a sensitivity of $274 \mathrm{~V} /(\mathrm{m} / \mathrm{s})$ in the range of $(20-80 \mathrm{~Hz})$ but these characteristics are not purely linear. Ideally the characteristics of MEMS seismic sensor should be independent of input vibrating frequency for the experiment reported in [2]. The purpose of this work is to study the limitations of the experimentation of MEMS based Seismic sensor such as frequency dependence and Artificial Neural Network based soft computing model have been proposed to compensate these errors. Compared to other seismometers relying on solid proof masses for environmental vibration monitoring, the electrochemical approach was reported to enable low-frequency vibration signal characterization [2], [3]. D. Chen, et al. reported micro electrochemical seismic sensor which have input vibrating frequency dependent characteristics. Figure 1 shows these characteristics. Input vibrating frequency [2], [3]. All of these reviewed work attempted to compensate different types of non-idealities of MEMS sensors however all these techniques require external hardware circuits or computer to compensate the errors. Our previous work focused on the development of Artificial Neural Networks based soft computing model [4] which results in frequency drift compensation with mean square error of 7.74 e-2. [4].Similar kind of compensation method applied to MEMS based gyroscope for minimizing angular rate error [5]

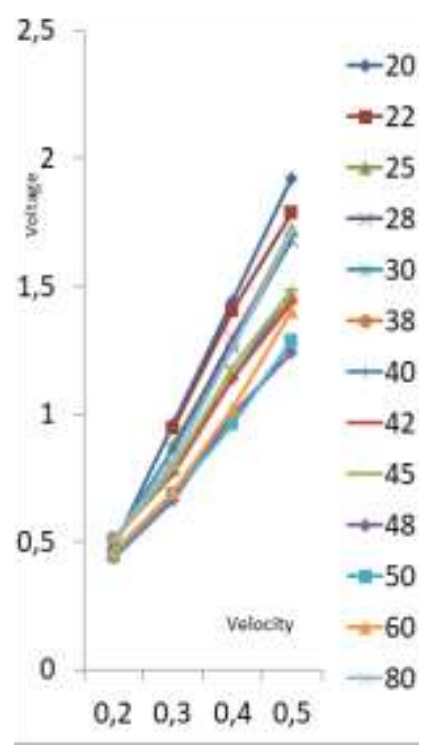

Figure 1. MEMS seismic sensor characteristics-velocity v/s output voltage of before vibrating frequency compensation 


\section{PROPOSED WORK}

\subsection{ANN Model Description}

Researchers have attempted for minimizing the non-idealities in MEMS based sensors like humidity sensors, porous silicon pressure sensors with external hardware or computer programme. But these applications are limited by more size and more power requirement which is not acceptable for highly compact circuits and system. Hence small size, less powered solution should be proposed for MSS. To characterize the parameters of commercially available MSS (MET2003), the devices were positioned on a vibrating table with the vibrating velocity and frequency adjusted systematically. Figure 1 records the output voltage of a MSS device as a function of the vibration velocity. It demonstrates the different output voltages for different vibrating frequencies which show linear relationship however these must be independent of input vibrating frequencies having purely linear relation. Hence this problem must be attempted by developing the interface circuit for drift compensation in output voltage. In our previous work, the feed forward ANN model with hyperbolic tan sigmoid activation function is trained with back propagation algorithm and the simulations are carried out in MATLAB, with ANN tool box. The frequency dependent output voltage is taken as input to ANN model which yields compensated output [4]. It results in frequency drift compensation with minimum mean square error (MSE) of 7.74 e-2. Figure 2. shows a neuron in ANN model. The input layer has two inputs and bias. Two neurons are selected for hidden layer. This work focused on FPAA based hardware implementation of input vibrating frequency independent interface circuit development.

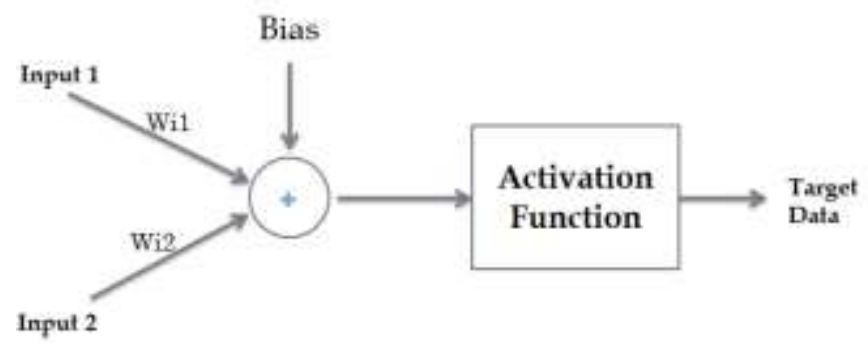

Figure 2. Basic single neuron model

\subsection{FPAA Structure}

Recently like FPGA in digital design, Anadigm provided Field programmable analog arrays (FPAA) with on board dynamic configuration capability gives designers user friendly platform in analog design. FPAA has fully differential architecture which is suitable and advantageous for low voltage analog signal processing. FPAA consist of four CAB blocks in which implementation of analog circuits is simpler, easier and less time taking. It uses the switched capacitance (SC) technology at the backend in which electrical charge through capacitor is controlled. Figure 3 shows the internal basic structure of the FPAA model AN231E04. The biggest advantage of FPAA is dynamic reconfiguration ability which provides on board parameter changing facility reducing time for development. It has seven configurable input-output cells and two dedicated output for interfacing. The main features including, low input offset through chopper stabilized amplifiers leading to better performance. The main four analog independent modules consisting of the lookup table, input and output interfaces having the analog functions crating facility. Look-Up Table (LUT) having 256 Byte capacities can be more suitable for sensor characteristics linearization and arbitrary signal generation. The typical frequency range for operation is from DC to $2 \mathrm{MHz}$ having Broadband Signal to Noise Ratio of $90 \mathrm{~dB}$ and Narrowband (audio) S/N ratio of 120dB [11]. 


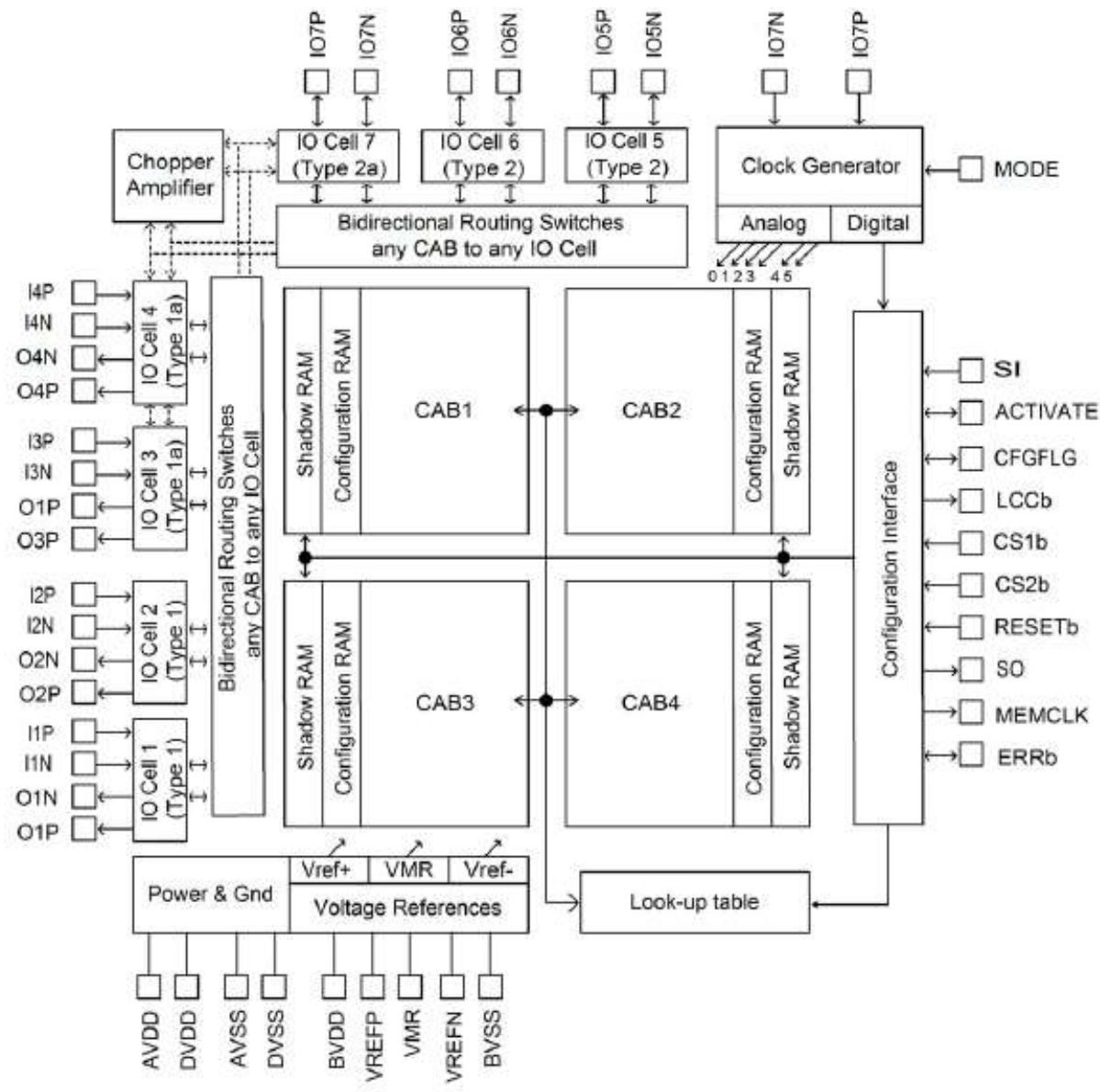

Figure 3. Internal block diagram of FPAA

\subsection{FPAA Based Implementation}

The vibrating frequency dependent output voltage $(0$ to $2.5 \mathrm{~V})$ of seismic sensor for vibrating velocity $(0$ to $0.5 \mathrm{~cm} / \mathrm{s}$ ) is used as input for FPAA based interface circuit which provides frequency independent output voltage. Figure 4 shows the basic working model of our research which has two parts consisting online sensor output processed by interface circuit and other having offline ANN model finding optimum parameters like weights and bias which are then taken into account while design of FPAA based interface circuit. Figure 5 depicts the single neuron implementation of FPAA based interface circuit in Anadigm Designer 2 simulator [12] which is then implemented in FPAA hardware development kit as shown in Fig.6. The one neuron is analysed in FPAA hardware which uses 3.3V as supply voltage for operation. The hyperbolic tan transfer function is implemented in look up table as shown in Figure 7.

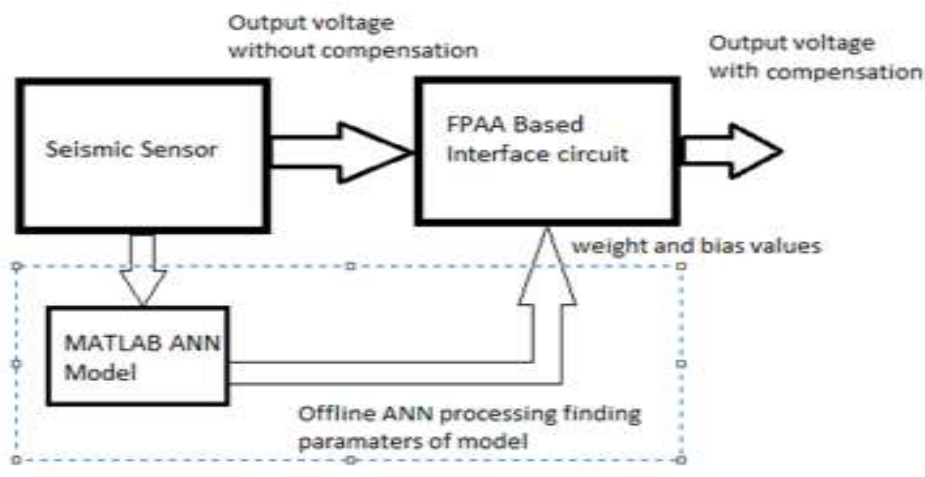

Figure 4. Block diagram of sensor with FPAA based Interface circuit 


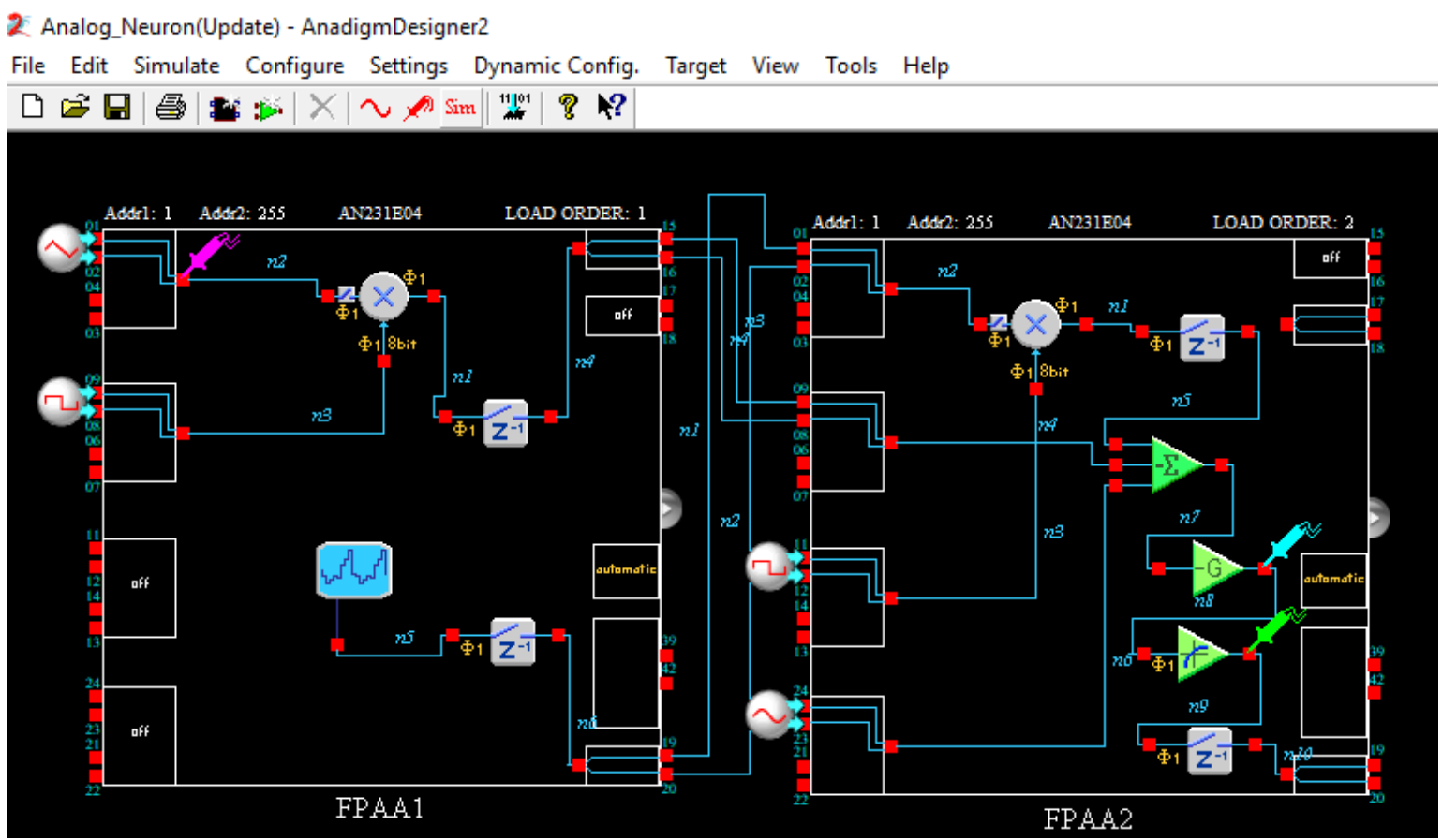

Figure 5. Implementation of FPAA based interface circuit in anadigm designer

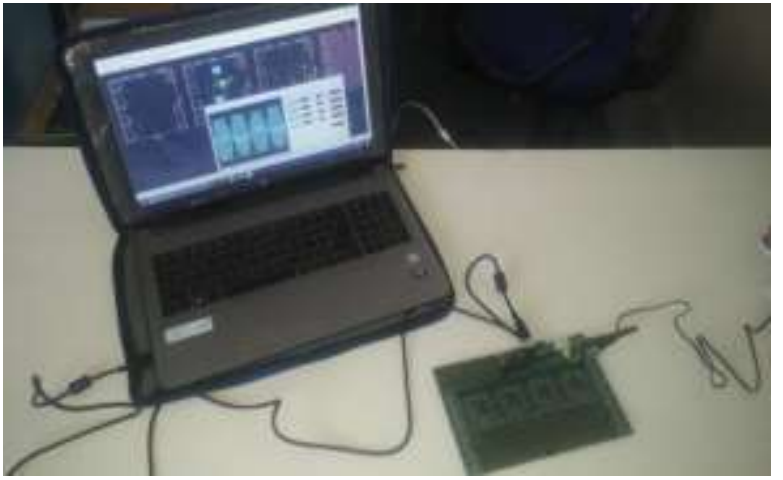

Figure 6. Implementation of FPAA based interface circuit

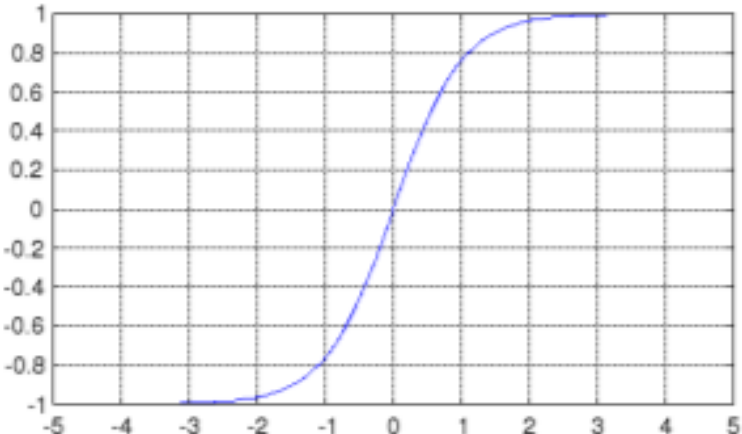

Figure 7. Hyperbolic tan transfer function implemented in look up table

\section{PERFORMANCE EVALUATION}

\subsection{Performance Metrics}

Error in percentage is calculated and analysed for the range of input velocity, 0 to $0.5 \mathrm{~cm} / \mathrm{s}$. The second metrics is used as the resource utilization in FPAA hardware which is also expressed in percentage.

\subsection{Discussion on Results}

In proposed system, the frequency dependent output voltage is taken as input to ANN model which yields compensated output. The input layer has two inputs and bias. For experimentation two neurons in hidden layer are used implement the circuit with simplicity. The selected method of training is back propagation algorithm and the simulations are carried out in MATLAB, with ANN tool box. Figure 8 shows minimized error with reference to velocity and Figure 9 shows the output voltage for different vibrating frequency, after applying ANN based compensation model in FPAA. Table 1 shows resource utilization 


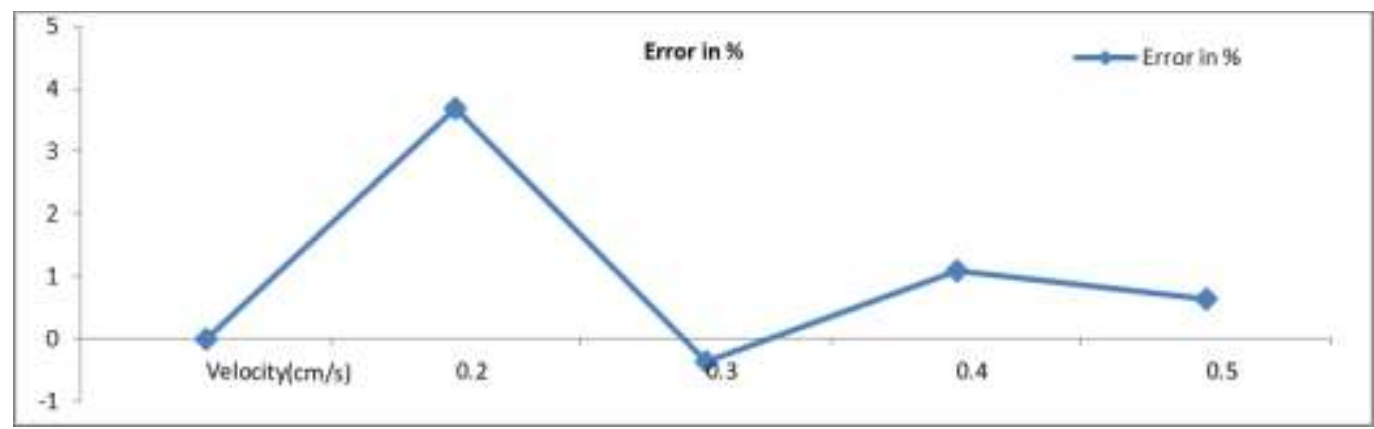

Figure 8. Error in percentage

Table 1. Resource Utilization

\begin{tabular}{ccccccccc}
\hline & \multicolumn{4}{c}{ FPAA1 } & \multicolumn{5}{c}{ FPAA2 } \\
Resources & C & Op & Co & SAR & C & Op & Co & SAR \\
\hline CAB 1 & 06 & 02 & 01 & 01 & 06 & 02 & 01 & 01 \\
CAB 2 & 06 & 02 & -- & -- & 08 & 01 & -- & -- \\
CAB 3 & -- & -- & -- & -- & 04 & 01 & -- & -- \\
CAB 4 & -- & -- & -- & -- & 06 & 01 & 01 & 01 \\
Total used & 12 & 04 & 01 & 01 & 24 & 05 & 02 & 02 \\
Available & 32 & 08 & 04 & 04 & 32 & 08 & 04 & 04 \\
Utilization in \% & 37.5 & 50 & 25 & 25 & 75 & 62.5 & 50 & 50 \\
Power & & $94.28 \mathrm{~mW}$ & & & $112.34 \mathrm{~mW}$ & \\
\hline
\end{tabular}

C: Capacitors, Op: No of Operational Amplifiers, Co: Comparators, SAR: Successive approximation resistors

Figure 9. shows the graphs of velocity and output voltage after compensation. FPAA based compensation circuit gives the error in frequency drift in the range of $3.68 \%$ to about $0.64 \%$ as compared to ANN simulated results in the range of $23.07 \%$ to $0.99 \%$. Figure 10 shows comparison of FPAA resource utilization

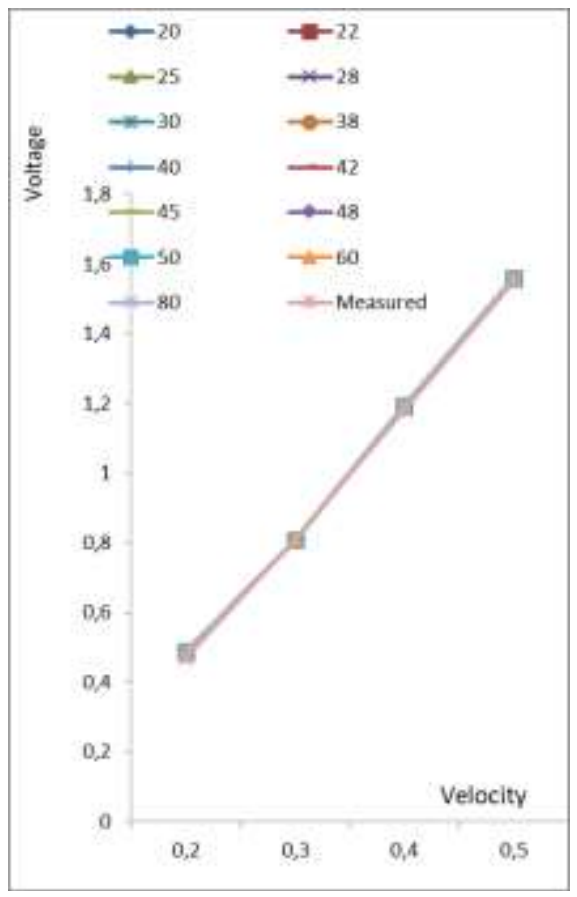

Figure 9. MEMS seismic sensor characteristics-velocity v/s output voltage of after vibrating frequency compensation 


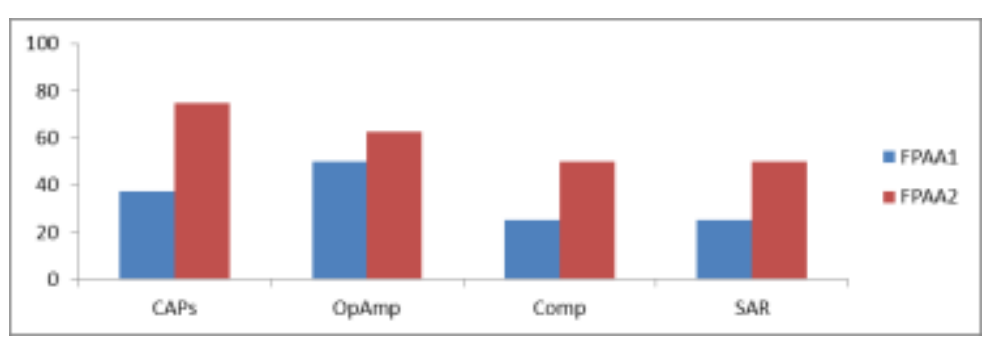

Figure 10. Comparison of FPAA resource utilization

\section{CONCLUSION AND FUTURE SCOPE}

In this paper we proposed input vibrating frequency dependent compensation model with minimum error and its implementation in the range of 0 to 0.5 velocities against the expected frequency independent compensation. FPAA based neuron implementation is proposed with quick prototype for interface circuit. This single neuron consumes of power of $206.62 \mathrm{~mW}$. The available resources are not fully utilized for one neuron implementation. Developed prototype gives advantages in terms of less time to implement, interactive hardware and simulation design environment however same circuit implementation in ASIC may take much time for implementation. Using this FPAA based compensation circuit, the error in frequency drift have been minimized in the range of $3.68 \%$ to about $0.64 \%$ as compared to ANN simulated results in the range of $23.07 \%$ to $0.99 \%$. As the proposed hardware consists all analog environment which removes the requirement of ADC and DAC reducing significant power and size of interface circuit. This work gives the SMART MEMS seismic sensor with reliable output and ANN based intelligent interface circuit implemented in FPAA hardware. Further work focuses on the CMOS based ASIC solution.

\section{ACKNOWLEDGMENT}

The authors extend their regards to SITRC Nashik, AVCOE, Sangamner and Research Promotion Scheme of BCUD, Savitribai Phule Pune University, India, for providing resources in research work. The authors declare that they have no competing interests.

\section{REFERENCES}

[1] Kaigham J. Gabriel, "Microelectromechanical Systems (MEMS) Tutorial”, IEEE International Test Conference, 1998, pp.432-441.

[2] D. Chen, et al., A micro electrochemical seismic sensor based on MEMS technologies, Sens. Actuators A: Phys. (2013), http://dx.doi.org/10.1016/j.sna.2012.12.041

[3] Guangbei Lia, Deyong Chena, Junbo Wanga, Chen Jiana, Wentao Hea, Yunjie Fana and Tao Denga, "A MEMS Based Seismic Sensor Using the Electrochemical Approach" Procedia Engineering 47 ( 2012 ) 362 - 365

[4] Ramesh Pawase, Dr.N. P. Futane, "Artificial Neural Networks Based Mathematical Modeling for Interface Circuit of MEMS Electrochemical Seismic Sensor for Frequency-Drift Compensation” International Symposium on Physics and Technology of Sensors (ISPTS-2)

[5] Ramesh Pawase, Dr.N. P. Futane,"Angular rate error compensation of MEMS based gyroscope using artificial neural network", International Conference on Pervasive Computing (ICPC), 2015,pp.1-4

[6] E.G. Bakhoum, M.H.M. Cheng, Frequency-selective seismic sensor, IEEE Trans-actions on Instrumentation and Measurement 16 (3) (2012) 823-829.

[7] Emil Dimitrov Manolov, Mihail Hristov Tzanov and Filip Todorov Koparanov, "FPAA Implementation and Investigation of Analog Neurons", ANNUAL JOURNAL OF ELECTRONICS, 2009, ISSN 1313-1842

[8] Anthony S. Deese, C. O. Nwankpa,"Design and Testing of Custom FPAA Hardware With Improved Scalability for Emulation of Smart Grids", IEEE TRANSACTIONS ON SMART GRID, VOL. 5, NO. 3, MAY 20141369.

[9] Dias Pereira JM, Postolache O, Silva Girao PMB, Cretu Mihai, "Minimizing temperature drift error of conditioning circuits using artificial neural networks", IEEE Trans Intrum Meas 2000, 49(5).

[10] N.P. Futane, S. Roy Chowdhury, C. Roy Chowdhury , H. Saha, "ANN based CMOS ASIC design for improved temperature-drift compensation of piezoresistive micro-machined high resolution pressure sensor", Microelectronics Reliability 50 (2010), pp.282-291

[11] N. P. Futane ,S. RoyChowdhury ,C. RoyChaudhuri ,H. Saha, ”Analog ASIC for improved temperature drift compensation of a high sensitive porous silicon pressure sensor," Springer, Analog Integr Circ Sig Process (2011) 67:383-393.

[12] Anadigm FPAA AN231E04 Datasheet Rev 1.1, http://www.anadigm.com/_doc/DS231000-u001.pdf

[13] Anadigm Designer®2, User Manual software version 2.4.0.5, http://www.anadigm.com/_doc/UM020800U001.pdf 